\title{
Development of a GEM-based TPC for H-dibaryon Search at J-PARC
}

\section{Hiroyuki Sako}

Japan Atomic Energy Agency

E-mail: hiroyuki.sako@j-parc.jp

\section{Sanghoon Hwang}

Japan Atomic Energy Agency

E-mail: shhwang@post.j-parc.jp

\section{Yudai Ichikawa}

Japan Atomic Energy Agency

E-mail: yudai@scphys.kyoto-u.ac.jp

\section{Kenlchi Imai}

Japan Atomic Energy Agency

E-mail: imai.kenichiljaea.go.jp

\section{Susumu Sato}

Japan Atomic Energy Agency

E-mail: susumu.sato@j-parc.jp

\section{Hitoshi Sugimura}

Japan Atomic Energy Agency

E-mail: sugimura.hitoshi@jaea.go.jp

\section{for the J-PARC-E42 Collaboration}

We developed a TPC using GEMs and a gating grid to search for the H-dibaryon at J-PARC with high rate hadron beams up to $10^{6}$ count per second $(\mathrm{cps}) / \mathrm{cm}^{2}$. The TPC consists of an octagonalshape drift cage filled with $\mathrm{Ar}-\mathrm{CH}_{4}$ (90:10) gas, and an end cap detector consisting of a gating grid, a triple GEM stack, and readout pads. The TPC is operated in the magnetic field of $1 \mathrm{~T}$. We built a small prototype TPC and performed tests. The hit position shift due to ion backflow was suppressed within $0.2 \mathrm{~mm}$ at the beam rate up to $5 \times 10^{5} \mathrm{cps} / \mathrm{cm}^{2}$. The hit detection efficiency was better than $90 \%$ up to the beam rate of $3 \times 10^{6} \mathrm{cps} / \mathrm{cm}^{2}$. The horizontal position resolution was improved by $40-50 \%$ in the magnetic field from 0 to $0.68 \mathrm{~T}$ at a laser test.

Technology and Instrumentation in Particle Physics 2014,

2-6 June, 2014

Amsterdam, the Netherlands 


\section{Introduction}

We developed a TPC to search for $\mathrm{H}$-dibaryon in ${ }^{12} \mathrm{C}\left(K^{-}, K^{+}\right) \Lambda \Lambda X$ reaction (the J-PARC E42 experiment [1]). Figure 1 shows a schematic view of the E42 TPC (HypTPC) [2]. The drift cage has an octagonal-prism shape of $58 \mathrm{~cm}$ diameter and the drift length (vertical) of $55 \mathrm{~cm}$, where the magnetic field of $1 \mathrm{~T}$ is applied in parallel to the drift field. The diamond target is positioned inside the drift volume with a cylindrical target holder. The end-cap detector consists of a gating grid and three-layer GEMs [3] (two GEMs with $50 \mu \mathrm{m}$ thickness and a GEM with $100 \mu \mathrm{m}$ thickness in the bottom). The configuration is adopted to suppress the ion backflow as much as possible to cope with field distortion. A high rate $K^{-}$beam of $10^{6} \mathrm{cps}$ is injected directly into the drift volume, and two $\pi^{-}$s and 2 protons are reconstructed. The $\Lambda \Lambda$ invariant mass resolution is required to be better than $2 \mathrm{MeV} / \mathrm{c}^{2}$. The hit position distortion due to ion backflow should be less than $1 \mathrm{~mm}$ in order not to deteriorate position resolution.
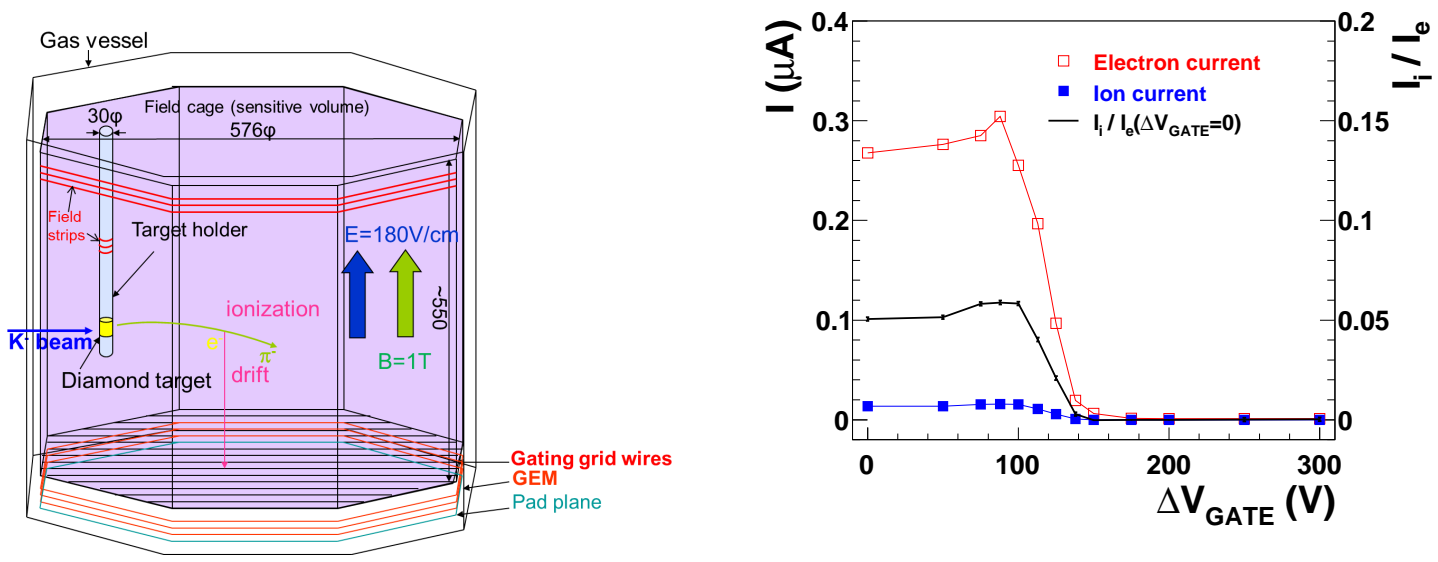

Figure 1: A schematic view of the E42 TPC Figure 2: The electron current (open squares) $(\mu \mathrm{A})$, (HypTPC) design.

the positive ion current (filled squares) $(\mu \mathrm{A})$, and the ion backflow fraction (thick line) as a function the gate voltage in $\mathrm{V}$, where the voltage of the $50 \mu \mathrm{m}$ GEM is kept to $325 \mathrm{~V}$

\section{Performance evaluation of the prototype TPC}

In order to evaluate performance required for E42, we built a small TPC with the transverse dimension of $10 \times 10 \mathrm{~cm}^{2}$ with the drift length of $20 \mathrm{~cm}$.

\subsection{Backflow measurement}

We measured the ion backflow using a ${ }^{90} \mathrm{Sr}$ source. Figure 2 shows the electron current and the ion current as a function of the gate voltage. At the gate voltage of 0 , the ion backflow fraction to the electron current is $4.5 \pm 0.2 \%$ at the GEM voltages of $325 \mathrm{~V}$ and $488 \mathrm{~V}$ for the $50 \mu \mathrm{m}$ GEMs and the $100 \mu \mathrm{m}$ GEM, respectively. The gain was $1.7 \times 10^{4}$. By applying the gate voltage, the ion 
backflow is suppressed further, and the ion backflow fraction at the gate voltage of $150-300 \mathrm{~V}$ is measured to be $0.00 \pm 0.04 \%$.

\subsection{Beam test}

In order to evaluate performances at high rates, we performed a test using a $400 \mathrm{MeV}$ proton beam. The horizontal resolution was measured to be $0.19-0.46 \mathrm{~mm}$ using TPC tracks at the drift length of 5-20 cm with 4-mm wide pads without the magnetic field. The expected horizontal position resolution at E42 with $1 \mathrm{~T}$ magnetic field is better than $0.3 \mathrm{~mm}$, which fulfills the requirement. Efficiency better than $90 \%$ was obtained at the beam rate up to $3 \times 10^{6} \mathrm{cps} / \mathrm{cm}^{2}$.

We measured the hit position distortion using external trackers as shown in Fig. 3. With the gate open, we observed the horizontal position shifts of $\pm 1.3 \mathrm{~mm}$ at the beam rate of $9.1 \times 10^{3} \mathrm{cps}$, while in the gate operation, it is suppressed within $\pm 0.2 \mathrm{~mm}$ at the beam rates up to $5 \times 10^{4} \mathrm{cps}$. The vertical distortion with and without the gate operation was within $\pm 0.2 \mathrm{~mm}$. The expected distortion at E42 was estimated using the measured data and the measured ion backflow, assuming linear dependence of the distortion on the ion current, to be $0.3 \pm 0.2 \mathrm{~mm}$ both in the horizontal and vertical directions.
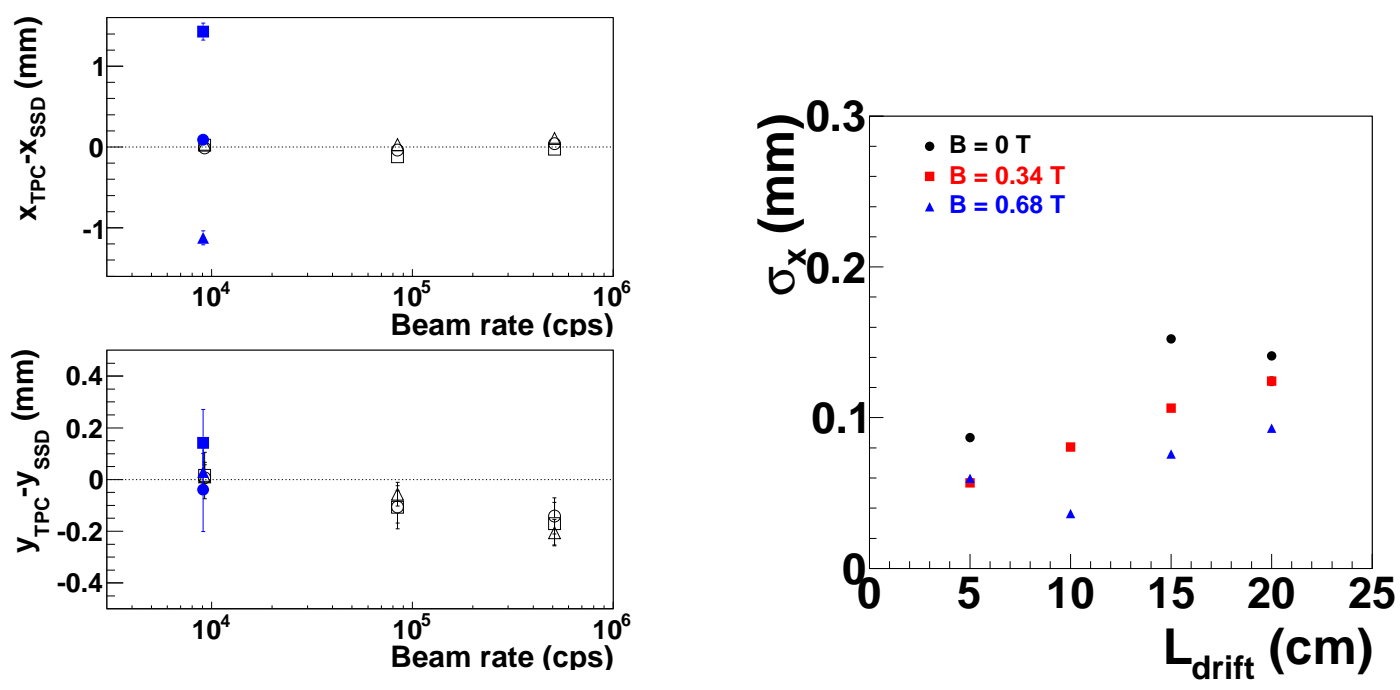

Figure 3: Horizontal (top) and vertical (bottom) clus- Figure 4: The horizontal resolution as a function of ter position distortion $(\mathrm{mm})$ with respect to those at a the drift length with the 3-mm wide pads at the maglow beam rate $\left(6.58 \times 10^{3} \mathrm{cps}\right)$ with the gate voltage netic fields of $0 \mathrm{~T}, 0.34 \mathrm{~T}$, and $0.68 \mathrm{~T}$.

of $150 \mathrm{~V}$ as a function of the beam rate for P-10. In the top (bottom) plots, circles, squares, and triangles are at $0,-4 \mathrm{~mm}$, and $+4 \mathrm{~mm}$ with respect to the beam center horizontal (vertical) position of $19.8 \mathrm{~mm}(143.3 \mathrm{~mm})$, respectively. Open symbols are at the gate voltage of $150 \mathrm{~V}$ and filled symbols are at $0 \mathrm{~V}$.

\subsection{Laser test with the magnetic field}

We performed a test in the magnetic field using a UV laser. We injected a horizontal YAG 
laser beam of $266 \mathrm{~nm}$ wavelength through quartz windows on the gas vessel. Fig. 4 shows the horizontal position resolution as a function of the drift length. We observed the reduction of the resolution by $40-50 \%$ at the magnetic field from 0 to $0.68 \mathrm{~T}$. Note that the resolution is smaller by $60 \%$ compared to that for a minimum ionizing particle due to a much larger number of ionized electrons.

\section{Conclusions}

In summary, we built a prototype TPC and evaluated performance required for the E42 experiment. We measured the backflow fraction of $5 \%$ with only the GEMs, and less than $\pm 0.04 \%$ also with the gating grid. The hit position distortion for $\mathrm{E} 42$ was estimated to be $0.3 \pm 0.2 \mathrm{~mm}$, within the requirement of $1 \mathrm{~mm}$. The efficiency was better than $90 \%$ at the beam rate up to $3 \times 10^{6} \mathrm{cps} / \mathrm{cm}^{2}$. The horizontal position resolution for E42 with $1 \mathrm{~T}$ magnetic field was estimated to be better than $0.3 \mathrm{~mm}$ from the measured resolution without the magnetic field. The horizontal position resolution was improved by $40-50 \%$ in the magnetic field of $0.68 \mathrm{~T}$ at the laser test. These prototype performance fulfil the requirements of E42. The E42 TPC has been designed based on the test results, and is currently under construction.

This work was supported by the Grant-in-Aid for Scientific Research on Innovative Area Grant Number 24105002 from MEXT, the Grant-in-Aid for Scientific Research (A) Grant Number 24244038 from JSPS, and the National Research Foundation of Korea (2012R1A2A1A01011926).

\section{References}

[1] J. K. Ahn and K. Imai, et al., J-PARC proposal P42.

[2] H. Sako, et al., JINST 9 (2014) C04009.

[3] F. Sauli, Nucl. Instrum. Meth. A386 (1997) 531. 